\title{
HEGEL Y LA ECONOMÍA POLÍTICA: CONTRIBUCIONES PARA UN DEBATE HISTÓRICO Y FILOLÓGICO
}

\author{
HEGEL AND POLITICAL ECONOMY: CONTRIBUTIONS FOR A HISTORICAL AND \\ PHILOLOGICAL DEBATE
}

\author{
Angelo Narváez León* \\ Pontificia Universidad Católica de Valparaíso, \\ Valparaíso - Chile. \\ Pablo Pulgar Moya \\ Universidad de Santiago, \\ Santiago - Chile. \\ Recibido julio de 2019/Received July, 2019 \\ Aceptado agosto de 2019/Accepted August, 2019
}

\begin{abstract}
RESUMEN
En el presente artículo presentamos el debate que se ha suscitado entre diferentes autores hegelianos acerca de la importancia, trascendencia y alcance epistemológico del concepto de economía política en Hegel. El debate acerca de las influencias económicas sobre la obra de Hegel oscila desde la posibilidad de influencias directas de lecturas de James Steuart y Adam Smith, hasta diálogos indirectos con ambos autores a través de lecturas de Locke, Hume y Ferguson. Aquí esbozamos la interpretación de exégetas como Georg Lukács, Norbert Waszek, José María Ripalda, Erzsébet Rózsa o Tetsushi Harada, quienes, entre otros autores, dan vida a un debate de por sí oscuro. La falta de escritos y la marginalidad de los textos de Hegel sobre el fenómeno de la economía política hacen del análisis un objeto difícil de asir. Aquí nos proponemos reconstruir historiográficamente los aspectos teóricos centrales de Hegel, a través obras, tendencias y traducciones, frente al fenómeno de la economía política. El análisis emprendido nos llevará a reconocer una diferencia en el tratamiento del tema entre el joven Hegel y el maduro, pero que comprende transversalmente la economía política no en la forma de elucubración acerca de los principios y axiomas que la componen, sino en las implicancias éticas que ella comporta. Para corroborar aquello, someteremos a juicio las diferentes hipótesis sobre la constitución económica en Hegel en su propia Filosofía del derecho, como también en sus respectivas lecciones.

Palabras Clave: Hegel, Economía Política, Ética, Mercantilismo.
\end{abstract}

\begin{abstract}
In this article we present the debate that has arisen among different Hegelian authors about the importance, transcendence and epistemological scope of the concept of political economy in Hegel. The debate about the economic influences on Hegel's work oscillates between the possibility of direct influences of readings of James Steuart and Adam Smith and indirect dialogues with both authors through readings of Locke's, Hume's and Ferguson's concepts of economy. Here we outline the interpretation of exegetes such as Georg Lukács, Norbert Waszek, José María Ripalda, Erzsébet Rózsa or Tetsushi Harada, who, among other authors, inject life to an already obscure debate. The lack of writings and the marginality of Hegel's texts on the phenomenon of political economy make the analysis an arduous task. Here we propose to reconstruct historiographically the central theoretical aspects of Hegel, through works, trends and translations, on the phenomenon of political economy. The analysis will lead us to recognize a difference in the treatment of the subject between the young and the old Hegel, but that transversally understands the political economy not in the form of elucubration about principles and axioms that compose it, but in the ethical implications
\end{abstract}

* Autor correspondiente / Corresponding author I Investigador postdoctoral: angelo.narvaez.1@ gmail.com 
that it entails. To corroborate that, we will put to judgment the different hypotheses about Hegel's economic position in his own Philosophy of law, as well as in their respective lessons.

Key Words: Hegel, Political Economy, Ethics, Mercantilism.

\section{INTRODUCCIÓN}

La relación de Hegel con la economía política ha sido puesta de relieve en múltiples ocasiones desde las más diversas perspectivas. Ahora bien, desde fines del siglo XIX, cuando Georgi Plejánov (2012) publicara su opúsculo con ocasión del $60^{\circ}$ aniversario de la muerte de Hegel, hasta el congreso celebrado en Urbino referido a la relación de la filosofía hegeliana con la globalización (Rinaldi \& Rossi, 2010), prácticamente todas las aproximaciones han compartido algunos aspectos comunes como, por ejemplo, una supuesta preminencia de los problemas económicos en un "joven" Hegel, en cuyo detrimento aparecerían posteriormente tópicos lógicos y políticos en el Hegel "maduro". Esta hipótesis, sin embargo, implica un problema ampliamente desatendido: si Hegel leyó la economía política clásica en su juventud, y además estos análisis tenderían a desaparecer en el periodo berlinés, entonces es de suponer que no habría mayores modificaciones en la conceptualización hegeliana de la economía política tras la publicación de la Fenomenología del espíritu. En el presente artículo trabajaremos una hipótesis diametralmente opuesta, pues no sólo sostenemos que las aproximaciones tempranas de Hegel a la economía política son cuantitativamente menores en relación a las aproximaciones posteriores, sino que, además, en el paso de Heidelberg a Berlín. Hegel modificará sustancial y cualitativamente su conceptualización. Esta modificación implica romper con la dualidad de un momento de "juventud" y otro de "madurez" en la filosofía hegeliana, para pensar los problemas de la economía política como una continuidad variable.

Para abordar este problema nos enfocaremos principalmente en el trabajo categorial que Hegel realiza en relación a la economía política durante toda su formación y desarrollo filosóficos. Así, dividiremos nuestra exposición en tres partes: i) primero, un análisis de la lectura de los Principles of Political Economy de James Steuart, y de cómo Hegel habría leído este trabajo a la luz de un prisma ético y filosófico, aunque categorialmente impreciso luego, ii) una caracterización filológica de la traducción que Hegel realizaría de la economía política en sus lecciones sobre derecho impartidas en Heidelberg y Berlín, donde encontraría una mayor precisión. Finalmente, iii), contextualizaremos el ambiente académico de las lecciones berlinesas para situar la conceptualización hegeliana en un largo proceso histórico de reformulación del pensamiento económico en la Alemania post-napoleónica. Este último punto nos permitirá abrir un problema que aquí sólo mentaremos, pero cuyo tratamiento no sólo ha sido desatendido por los estudios especializados, sino que además ha sido profundamente desconocido.

Metodológicamente vincularemos estos tres apartados a partir de un análisis filológico comparativo a partir de los manuscritos hegelianos, como también de las grandes tesis analíticas de los estudios especializados. Abordando el problema de esta manera, podremos seguir con el mayor detalle posible el modo en que la economía política fue adquiriendo y modificando su forma en la filosofía hegeliana desde el periodo jenense al periodo berlinés.

\section{HEGEL Y LA ECONOMÍA POLÍTICA EN EL FIN DE SCİ̀CLE ALEMÁN}

Hacia fines del siglo XVIII y comienzos del siglo XIX, los economistas escoceses fueron recibidos con especial entusiasmo en los espacios universitarios continentales en detrimento, por cierto, de la influencia que ejercieran alguna vez las tesis de la fisiocracia francesa. En Alemania particularmente, sostiene Keith Tribe (1988), los Principles of Political Economy de James Steuart, eran "hasta la última década del siglo XIX (...) más conocidos y más frecuentemente citados que La riqueza de las naciones de Smith" (p. 143). Esta preponderancia, según argumenta Ramón Tortajada (1999), se debió al menos a tres factores: i) de una parte, Steuart pasó algunas temporadas en Alemania, llegando a escribir parte de sus Principles en Tübingen; ii) de otra, esta estadía implicó que no pocos cameralistas alemanes asumieran las posiciones económicas de Steuart como una renovación necesaria de las proposiciones 
categoriales y de las prácticas económicas "más atrasadas" de Alemania; iii) y, finalmente, entre 1769 y 1772 se publicó en Tübingen una traducción que rápidamente se convirtió en prácticamente el único material de la Ilustración económica anglo-escocesa disponible en Alemania. Sin embargo, este escenario inicialmente dominado por Steuart daría paso hacia fines del siglo XVIII a un acelerado proceso de transformación académica de la economía política en Alemania a partir de los análisis propuestos por Adam Smith.

La primera traducción alemana de La riqueza de las naciones fue publicada en Leipzig en dos volúmenes entre 1776 y 1778. La edición, traducida como Untersuchungen der Natur und Ursachen von Nationalreichthümen, y realizada por Johann Friedrich Schiller y Christian August Wichmann, fue objeto de tempranas críticas publicadas en el Göttinger Anzeigen von gelehrten Sachen y en la Allgemeine deutsche Bibliothek. Una de las críticas más populares fue la publicada entonces anónimamente por J. G. H. Feder el 10 de marzo de 1777 en el Göttinger Anzeigen. Al momento de publicarse las críticas de Feder, Göttingen formaba parte del Electorado de Brunswick-Lüneburg (Hannover) y, por tanto, dependía políticamente de las disposiciones emanadas del trono de Jorge III de Inglaterra. La Universidad de Göttingen había sido fundada en 1737 por el abuelo de Jorge III, Jorge II, y muy prontamente se transformaría en un centro de difusión científica que atraería a estudiantes posteriormente determinantes de las reformas alemanas de comienzos del siglo XIX. Así, no sólo Wilhelm von Humboldt, sino también funcionarios como K. A. von Hardenberg y K. F. vom Stein pasarían por las aulas de Göttingen en las cuales Feder discutiría introductoriamente las hipótesis económicas de Smith en explícita oposición a los trabajos de Steuart. En palabras de Klaus Epstein (1966) "todos los profesores importantes de Göttingen - Feder, Pütter, Schlözer, Sartorius, et cetera [sic]_-" se vieron influidos por la nueva economía política, haciendo de ésta, junto a Königsberg, uno "de los grandes centros académicos que esparcieron las ideas de Smith" (p. 180).

Georg Sartorius, quien recensionaría también la traducción de Schiller para el Göttinger Anzeige, publicaría en 1796 su Handbuch der Staatwirtschaft zum Gebrauche bey akademischen Vorlesungen, nach Adam Smith's Grundsätzen ausgearbeitet, trabajo a partir del cual, por ejemplo, Goethe se introduciría en el estudio de la economía política (Knortz \& Laudenberg, 2014, p. 7). Luego, entre 1800 y 1802, August F. Lueder publicaría su Über Nationalindustrie und Staatswirtschaft. Nach Adam Smith bearbeitet, y en 1804, F. Weber haría lo mismo con su Systematisches Handbuch der Staatswirtschaft. Todos estos esfuerzos contribuyeron a la maximización de la presencia de Adam Smith en Alemania; sin embargo, ninguno de ellos tuvo tanta repercusión como la traducción de La riqueza de las naciones publicada por Christian Garve entre 1794 y 1796, publicada como Untersuchung über die Natur und die Ursachen des Nationalreichthums (Lai, 2003). Este escenario de debate y transformación de las ciencias económicas modernas es el que enfrenta Hegel en relación a la economía política.

Georg Lukács, uno de los primeros especialistas en hacer de la economía política un problema sistemático de investigación en la filosofía hegeliana, sitúa el punto de inflexión en la formación económica de Hegel en la lectura de Adam Smith a través de la traducción de Christian Garve (Lukács, 1963), y a la misma tesis adscriben posteriormente James Henderson y John B. Davis (1991). Terry Pinkard sostiene la posibilidad que Hegel se haya familiarizado con las proposiciones económicas de Smith ya desde 1796, a partir de la edición inglesa publicada en Basilea en 1791. Pinkard llegará a sostener la improbabilidad que Hegel no haya trabajado el texto, atendiendo a las condiciones económicas que sustentaban su vida cotidiana en Berna y al "despilfarro" que significaba en 1796 darse el lujo de adquirir un libro por puro capricho (2002). Norbert Waszek va un paso más allá al sostener que Hegel habría adquirido la edición de Basilea atendiendo a las críticas esgrimidas por Feder a la traducción de Schiller (1988). En cualquier caso, ninguno de estos recorridos biográficos permite dirimir con claridad la antecedencia de Steuart o Smith en la formación económica hegeliana.

Desde la publicación de Hegels Leben, la biografía preparada por Karl Rosenkranz en el marco de la edición de las Hegels Werke. Vollständige Ausgabe durch einen Verein von Freunden des Verewigten, prácticamente todas las investigaciones que han tenido por objeto caracterizar el lugar y la función de la economía política en la filosofía hegeliana, han enfrentado un problema al momento 
de dar un sentido disciplinar al relato biográfico. De acuerdo a Rosenkranz,

Todos los pensamientos de Hegel sobre la esencia de la sociedad burguesa, sobre las necesidades y el trabajo, sobre la división del trabajo y fortuna de los estamentos, asistencia social y orden público, impuestos, etc. terminaron concentrándose en un comentario en forma de glosas a la traducción alemana de la Economía política de Steuart. (Hegel, 2003, p. 258).

En el comentario, escrito entre febrero y mayo de 1799, insiste Rosenkranz, James Steuart aparecería como un "partidario del mercantilismo", sin embargo, "con noble pathos, con abundancia de interesantes ejemplos, Hegel luchó contra lo muerto de ese sistema, tratando de salvar la sensibilidad [Gemüth] del hombre en medio de la competencia como en el mecanismo del trabajo y del comercio" (Hegel, 2003, p. 258). Robert C. Salomon (1983) relativiza el relato biográfico de Rosenkranz al sustituir la influencia del pensamiento económico de Steuart principalmente por los de Adam Ferguson y, secundariamente, por los de John Locke y David Hume (p. 111). El problema con la tesis de Salomon estriba en que sabemos justamente a partir de los fragmentos tempranos que el énfasis de Hegel en sus lecturas de Ferguson, Locke y Hume está puesto en los problemas políticos, administrativos y constitucionales antes que en aquellos referentes a la economía política. De una u otra manera, disputar la antecedencia de tal o cual autor en la formación del pensamiento económico hegeliano parece llevarnos indefectiblemente a círculo vicioso. Ahora bien, si se toma el comentario perdido no como un criterio de antecedencia sino como un problema en sí mismo, el horizonte analítico es harto más provechoso, especialmente si atendemos dos problemas en particular: i) el uso del término "mercantilismo" y, ii) el sentido de la "sensibilidad".

Primero, que en el comentario perdido el mercantilismo haya sido objeto de crítica, haría suponer que Hegel habría tomado tempranamente una posición en favor de Smith en detrimento de Steuart. El juicio de Adam Smith sobre el mercantilismo en La riqueza de las naciones no es, ni por lejos, benévolo. Para Smith "el sistema mercantil no ha sido muy favorable para la renovación del cuerpo de la población, (o) para el producto anual de la tierra y del trabajo de una nación”; y, así “(...) tampoco parece haber sido más favorable para la formación de la soberanía, al menos hasta que ésta dependa de los impuestos a las mercancías" (Smith, 1981, p. 881). Smith (1981), empeñado en la renovación de la economía inglesa, llegaría a tildar al mercantilismo de sofistería (p. 927).

Ahora bien, en su edición de La riqueza de las naciones, Christian Garve, tradujo "comercial, or mercantile system" por "Handel = oder kaufmännische System" (Smith, 1799, p. 3), hecho que dio paso a una confusión popularizada por Paul Chamley (1962), quien sostiene que la inclusión del término Merkanilsystem, en vez de kaufmännische System, correspondería a un exceso del relato biográfico de Rosenkranz. Sin embargo, Chamley aparentemente desconoce, o desatiende, que hacia 1799 Hegel tenía a disposición sólo la edición de Basilea, y que sólo hacia 1803 se haría de una copia de la traducción de Garve, por lo que no resulta improbable que Rosenkranz haya tomado el término del manuscrito del propio Hegel. Lejos de constituir una discusión exclusivamente filológica, la posición que se adopte en relación al sentido del Merkantilsystem, supone una posición relativa al segundo punto en cuestión.

Si Hegel era consciente del sentido smithiano del sistema mercantil al momento de redactar su comentario a los Principles de Steuart, entonces la "sensibilidad [Gemüth] del hombre" debía, en principio, ser salvada justamente del mercantilismo y no de la nueva economía política propuesta por Smith. El problema no es tan claro como parece, pues implica dirimir entre una posición crítica del mercantilismo en favor de la nueva economía, o en favor del cameralismo típicamente alemán, tesis a la cual adscribe Claudio Mario Aliscioni (2010). Como bien ha notado Norbert Waszek (1988), rescatando las observaciones de Otto Pöggeler, el concepto de Gemüth no implicaría estrictamente un sentido de sensibilidad, sino más bien una precisión en relación a la totalidad [Ganzheit] del hombre. Para Waszek, este es un término técnico propio de fines del siglo XVIII que, tomado de Meister Eckhart y Jakob Böhme, sería popularizado por Schiller en sus Cartas sobre la educación estética del hombre, con las cuales Hegel estaba perfectamente familiarizado (p. 144). Si seguimos esta línea sería necesario asumir que la crítica de Hegel al mercantilismo se habría construido a partir de una defensa del cameralismo económico-político 
consuetudinario del Sacro Imperio, algo más caro al primer Romanticismo, tesis ampliamente desarrollada por Tetsushi Harada (1987, p. 110).

El problema con las tesis de Aliscioni y Harada radica en que Hegel ya había criticado los fundamentos filosóficos y las consecuencias sociales del cameralismo consuetudinario en un texto redactado un año antes del comentario a Steuart, es decir, en 1798: Da $\beta$ die Magistrate von den Bürgern gewählt werden müssen. No hay, por tanto, buenas razones para suponer que Hegel haya criticado el derecho político consuetudinario en orden a defender, frente al mercantilismo, una posición cameralista consuetudinaria. Otra posibilidad, que desarrollaremos a continuación, es que Hegel incluso habiendo leído los trabajos de Smith desde 1796, asociara tanto el mercantilismo como la nueva economía política a los problemas de una hipotética pérdida de la unidad atravesada por los principios de la división social del trabajo y la competencia.

En La riqueza de las naciones, Adam Smith (2004) define con claridad que la economía política, "considerada como una rama de la ciencia del hombre de Estado o legislador", es una ciencia que tiene por finalidad, de un lado, "conseguir un ingreso o una subsistencia abundantes para el pueblo" y, de otro, "proporcionar al Estado o comunidad un ingreso suficiente para pagar los servicios públicos" (p. 539). Steuart por su parte, también entiende la economía política como una ciencia cuyo principal objetivo es,

\begin{abstract}
to secure a certain fund of subsistence for all the inhabitants, to obviate every circumstance which may render it precarious; to provide every thing necessary for supplying the wants of the society, and to employ the inhabitants (supposing them to be free-men) in such a manner as naturally to create reciprocal relations and dependencies between them, so as to make their several interests lead them to supply one another with their reciprocal wants. (1767, p. 6).
\end{abstract}

En principio, la primera definición de economía política propuesta por Hegel no difiere de las propuestas de Steuart y Smith, aunque pone el énfasis en la interdependencia de los actores económicos antes que en los mecanismos particulares que darían forma, por ejemplo, a los ingresos nacionales. En 1802, sostiene Hegel (1979) que “en cuanto ciencia, el sistema de la llamada economía política [politische Ökonomie]", tiene por objeto el análisis de la "dependencia recíproca universal en la perspectiva de las necesidades físicas, así como en la del trabajo y la acumulación" (p. 60).

Si tomamos esta primera definición como criterio para precisar el proceso formativo de la economía política en el pensamiento hegeliano, no es del todo posible salir del problema precedente, pues como han notado José María Ripalda (Hegel, 2006, p. 327) y Fausto Barcella (1975, p. 101), el recorrido argumentativo ensayado en el Sistema de la eticidad, de 1803, y en el Systementwürfe, de 1803/4, Hegel tomaría, por ejemplo, el sentido de las categorías "valor", "dinero" y "trabajo" no de la obra de Smith, sino de la noción de "trabajo útil" de Steuart. Ripalda insiste sobre este punto al mostrar la imprecisión con la cual Hegel refiere la obra de Smith en el contexto del Systementwürfe:

En una manufactura inglesa una aguja pasa por las manos de 18 trabajadores a cada uno le corresponde un aspecto particular del trabajo y sólo ése. Uno sólo tal vez no pudiera hacer 20, ni siquiera 1. Esos 18 trabajos repartidos entre 10 hombres producen 4.000 diarias; pero si el trabajo de éstos 10 lo realizan 18 , serán 48.000 por día. (Hegel, 2006, p. 326).

El problema, concluirá Ripalda en su estudio filológico, estriba en que "las referencias numéricas de Smith están completamente tergiversadas: Smith dice que una libra contiene 4.000 agujas y que 10 obreros realizando 18 operaciones en total producen en un taller que él visitó 48.000 agujas al día" (Hegel, 2006, p. 327). Entonces, si bien es cierto que hay una preocupación temprana por la economía política en el pensamiento hegeliano, esta radica en un análisis de las consecuencias éticas posibles antes que en una conceptualización precisa de los principios económicos de la modernidad, pues, como ha notado Jorge Dotti (1983), el Hegel de los manuscritos jenenses acentúa la importancia del "momento económico, porque ve en él uno de los polos en torno al cual giran los elementos más hostiles a someterse a la conciliación y que plantean así el dilema que él siente destinado a resolver con su filosofía" (p. 136). Es por esta razón que, como ha puesto de relieve David DeGrood (1979), las imprecisiones estrictamente económicas de Hegel, hacia 1803/04, 
pasan a un segundo plano en la medida que el interés filosófico habría versado prioritariamente sobre la paradoja ética de las sociedades comerciales mercantiles y/o capitalistas. Para DeGrood, en última instancia, no se trata de dirimir entre una posición en favor o en contra del mercantilismo o del capitalismo, de Steuart o de Smith, sino de la posición ética de Hegel frente a los procesos comunes de industrialización de ambos sistemas económicos, y sus consecuencias empíricas como pauperización de la población. Al caso, podemos anticipar, para el Hegel jenense no se trata tanto del debate interno de la economía política como de la situación y condición de la clase trabajadora en Inglaterra. Así también lo afirma Rosenkranz en su relato biográfico: "En lo que toca al lucro y la propiedad, le fascinaba sobre todo Inglaterra", pues en ningún otro país de Europa se habían "desarrollado las formas de lucro y propiedad con tanta variedad como precisamente en Inglaterra, y a esa variedad le corresponde en las relaciones humanas una diversidad igualmente rica" (Hegel, 2003 , p. 257). En este sentido, Hegel reconoce la economía política, en cuanto práctica, como base del aspectos sociales y políticos precisos, razón por la cual, concluye Rosenkranz, Hegel siguió con especial interés "las sesiones del parlamento sobre la tasa de los pobres [Armentare] como la limosna con que la aristocracia de sangre y de dinero esperaba aplacar la excitación de las masas hambrientas" (Hegel, 2003, p. 257). Para el Hegel jenense el problema filosófico no estriba, en estricto rigor, en la existencia empírica de la riqueza y la pobreza, sino en la maximización de ambas dimensiones transidas por una formación socioeconómica que comenzaba a abrirse paso sistemáticamente en Inglaterra. En esta formación socioeconómica, insistirá Hegel en el Sistema de la eticidad, "el vínculo [Band] absoluto del pueblo, lo ético [das Sittliche], ha desaparecido, y el pueblo ha quedado disuelto" (1992, p. 186).

Ahora bien, la disolución del vínculo ético, así como los alcances del debate hegeliano sobre la pobreza material, son dos problemas que escapan al foco que hemos propuesto en esta investigación, situada más bien en el largo recorrido del concepto de economía política en la filosofía hegeliana. Llegados a este punto, en el orden a establecer un criterio claro para distinguir los principios económicos que animarían el lenguaje y la crítica hegeliana en su comentario a los Principles de
Steuart, sostenemos que dentro de los márgenes del periodo jenense, la conceptualización hegeliana de la economía política es, al menos, imprecisa en términos disciplinares. Sin embargo, en términos filosóficos, Hegel concluye su análisis en términos éticos que, si bien implican una dimensión antecedente económica, no constituye un argumento de lo que entre los siglos XVIII y XIX se entendería como un problema estrictamente económico. Lo que debemos atender a continuación entonces, es el segundo momento de encuentro de Hegel con la economía política para analizar sus variantes y alcances.

\section{LA ECONOMÍA POLÍTICA EN HEIDELBERG Y BERLÍN}

Tras el periodo jenense Hegel no retomará los problemas de la economía política al menos hasta 1817 cuando, de acuerdo a Petry (1976) y Waszek (1984, 1985, 1986), en el contexto de preparación de la primera lección sobre filosofía del derecho aparezcan las lecturas del Morning Chronicle, del Edinburgh Review y del Quarterly Review. La importancia de este punto de inflexión estriba en que, en la primera edición de la Enciclopedia de las ciencias filosóficas, la economía política no cumple ninguna función. Para el Wintersemester de 1817/18, Hegel (1983, p.117) distingue dos formalizaciones de la economía política: de una parte, elabora la necesidad racional del estudio de una Staatsökonomie, que tendría por objeto el análisis del comportamiento de la contingencia material de la realidad en relación a la totalidad de la sociedad; y, de otra, la necesidad de distinguir esa Staatsökonomie de la especificidad financiera inmediatamente de una Staatswirtschaft, la cual correspondería a la esfera del gasto nacional determinado por un Estado particular.

Esta distinción entre Staatsökonomie y Staatswirtschaft, siguiendo la hipótesis propuesta por Rózsa (2007), implica reconocer en el Hegel de Heidelberg y Berlín la presencia de una Wirtschaftphilosophie antes que de una Ökonomie, pues a la filosofía no le correspondería, por definición, la dilucidación de las formas económicas específicas de acuerdo a las cuales un Estado debe comportarse comercial y tributariamente, sino que, y por el contrario, sólo la representación comprensiva de la relación del comportamiento económico de la contingencia de la realidad. Distinción que, por lo demás ausente en los trabajos 
previos a 1817, cumplirá ahora una función de criterio demarcatorio de los alcances filosóficos de la economía política, en el orden a subvertir las aproximaciones del periodo jenense en el cual nos enfrentamos al debate sobre la posibilidad de situar la reflexión crítica hegeliana en relación al mercantilismo desde el horizonte del cameralismo o desde la economía política moderna.

Por otra parte, recién llegado a Berlín, Hegel enviaría una misiva al Ministerium des Unterreichts en la cual abogaría por la necesidad de una publicación científica "en que todas las ciencias [Wissenschaften] y ramas [Zweigen] especializadas [..., que representen] el interés general que la política de nuestro tiempo [Zeitpolitik] requiera" (1969, XI, p. 11) encontraran un espacio público de debate y discusión. Esta misiva expresa dos aproximaciones notables a la representación hegeliana de la economía política, pues de una parte, distingue entre las ciencias generales, entre ellas la Kameralwissenschaft y las ciencias de perspectiva filosófica [philosophische Gesichtspunkt], donde junto a los sistemas de filosofía natural y la teología se encuentra la Staatsökonomie. Es decir que Hegel no niega la posibilidad de una Kameralwissenschaft, pero entiende que esta se encuentra determinada por una significación puramente histórica, mientras la Staatsökonomie estaría determinada por una significación filosófica y científica.

Tras la formulación propuesta en Heidelberg y la distinción ensayada en la misiva berlinesa, Hegel presentará en 1820 una nueva definición de la economía política en el marco de la publicación de los Grundlinien der Philosophie des Rechts. Aquí Hegel sostendrá que "La economía política [Staatsökonomie] es la ciencia [...] que explica la relación y el movimiento de las masas en su determinación cualitativa y cuantitativa y en su desarrollo" (1993, p. 626). Esta definición, que sintetiza la discusión sobre la Staatsökonomie y la Staatswirtschaft de 1817/18, será replicada por Hegel a lo largo de todas sus lecciones sobre derecho impartidas posteriormente en Berlín hasta 1831. Esta definición de la economía política difiere por mucho de la ofrecida por Adam Smith en La riqueza de las naciones, como también de la ofrecida por James Steuart en sus Principios de economía política. Ahora bien, al ofrecer su definición de la economía política en el contexto de los Grundlinien de 1820, Hegel suprime sus referencias a Steuart y, en su lugar, posiciona los trabajos de David Ricardo y Jean-Baptiste Say. Sin embargo, las definiciones de la economía política ofrecidas en los Principios de economía política y tributación, como en el Tratado de economía política, respectivamente, no sólo difieren de la definición propuesta por Adam Smith, sino que —y más importante aún- en poco coinciden con la formulación hegeliana. David Ricardo sostiene que la economía política determina las leyes que regulan la distribución de la riqueza (1971, p. 3), y en una perspectiva análoga Say afirma que "La Economía política no considera la agricultura, el comercio y las artes sino por la relación que tienen con el aumento o la disminución de las riquezas, y de ningún modo en sus métodos o formas de ejecución" (2001, p. 21). Destutt de Tracy, de quien Hegel poseía una copia del Traité d'Économie politique, define la ciencia económica como la necesidad de "examiner quelle est la meilleure manière d'employer toutes nos facultés physiques et intelecctualles, à la satisfaction de nos divers besoins" (1823, p. II). Si bien esta definición se aproxima a la propuesta elaborada por Hegel en los Grundlinien, el Traité data de 1823 y la formulación hegeliana, según ya hemos dicho, no variará entre 1820 y 1831 . Con todo, las definiciones de la economía clásica no podrían coincidir con el contexto analítico de los Grundlinien, pues de hacerlo Hegel estaría abogando por una Staatswirtschaft "edificante" y no por una Staatsökonomie reflexiva. En este sentido se equivoca Norbert Waszek al sostener que en el lenguaje hegeliano Nationalökonomie, Staatsökonomie, Politische Ökonomie y Staatswirtschaft representan sinónimos sin matices $(2011$, p. 60), sobre todo cuando con toda claridad hay en el tránsito de la Staatswirtschaft a la Staatsökonomie una pretensión con conceptualizar cada vez con más precisión el sentido de la nueva economía política inglesa (cf. Priddat, 1990, p. 13). Con todo, es preciso ahora analizar entonces las posibles variaciones de esta conceptualización en momentos posteriores a la formulación de 1820 .

\section{HEGEL Y LA ECONOMÍA POLÍTICA POSTERIOR A 1820}

Hemos insistido en que, en su generalidad, la definición de economía política propuesta por Hegel en los Grundlinien der Philosophie des Rechts no sufriría mayores modificaciones en las 
lecciones berlinesas posteriores. Sin embargo, es necesario precisar algunas dimensiones y debates sobre esta posibilidad. David MacGregor, por ejemplo, sostiene que las críticas de Hegel a Adam Smith provendrían de una lectura temprana de los textos de David Ricardo. Si bien Hegel refiere los Principles en los Grundlinien, su edición personal de la obra de Ricardo correspondía a la traducción francesa de Francisco Solano Constâncio, que se publicó en 1819 junto con los comentarios crítica de Jean-Baptiste Say. Si bien es improbable que Hegel trabajara los Principles de Ricardo 1819 como para elaborar una crítica a Smith ese mismo año, en el contexto de redacción de la Filosofía del derecho, es posible que en el texto de 1820 la mención a Say provenga de manera indirecta a través la edición crítica preparada por Solano Constâncio. MacGregor lleva más allá su intuición, e incluso llega a sostener que la teoría hegeliana de la propiedad privada podría haber tenido algún tipo de influjo directo o indirecto de los trabajos de Thomas Hodgskin (1996, p. 138). El problema con esta hipótesis es que los trabajos de Hodgskin, Labour Defended against the Claims of Capital y Popular Political Economy, datan de 1825 y 1827 respectivamente, años en los que Hegel no sólo no modificaría los aspectos centrales de su conceptualización de la economía política en general, y de la teoría de la propiedad privada en particular, sino que además son los años en los que las lecciones berlinesas sobre derecho estaban bajo el cuidado de Eduard Gans.

Tras las lecciones del Wintersemester de 1824/25, Eduard Gans se haría cargo de la filosofía hegeliana del derecho hasta 1830/31. Hegel moriría tras impartir las primeras lecciones del Wintersemester de 1831/32, llegando sólo a trabajar los primeros conceptos introductorios, por lo que no sabemos filológicamente qué modificaciones podrían haber sido introducidas en relación a la conceptualización de la economía política que Hegel haya trabajado individualmente entre 1825 y 1831. Lo interesante de este periodo es que, por ejemplo, el mismo Gans introduce conceptos ausentes en la formulación hegeliana tales como Merkantilsystem, physiokratische System e Industriesystem, precisando una terminología algo ausente en la propuesta hegeliana. También discutirá explícitamente las tesis de autores como Colbert, Malthus, Galli, Raus, Machus y Nebenius, todos ausentes en los Grundlinien y en las lecciones berlinesas (Gans,
2005, p. 164). Gans también trabajó las tesis de Saint-Simón, a pesar de que Hegel sólo llegara a mencionarlo una vez en el Wintersemester de $1825 / 25$ (1973, IV, p. 520).

Paralelamente, así como en Göttingen a comienzos del siglo XIX, en el Berlín post-napoleónico la economía política tuvo un momento de auge y reformulación. En 1818, Johann Friedrich Gottfried Eiselen publicó su Über die Grundzüge der Staatswirtschaft oder der freien Volkswirtschaft und der sich darauf beziehenden Regierungskunst. Eiselen impartió además, entre 1817 y 1821 —año en que se trasladaría a Breslau-, lecciones sobre Finanzwissenschaft y Nationalökonomie. También en 1818, Johann Gottfried Hoffmann publicó su Übersicht der Bodenfläche und Bevölkerung des preußischen Staats y, en 1821, su Beiträge zur Statistik des preußischen Staats, trabajos donde discutirá abiertamente las hipótesis demográficas y económicas de Malthus. Friedrich Christoph Förster, amigo de Hegel y miembro de Verein tras la muerte de este, impartió lecciones sobre Allgemeine Erdkunde. Publicó en 1818 su Grundzüge der Geschichte des preußischen Staates, y entre 1820 y 1822 su Handbuch der Geschichte, Geographie und Statistik des preußischen Reichs, donde también discutiría la relación entre economía y demografía.

La productividad de Eduard Gans es especialmente prolífica en este periodo: en 1827 publicó Das Englische Staatsrecht in seiner historischen Entwickelung, en 1828, Geschichte der neuesten Zeit von 1789 an, in besonderer Beziheung aur öffentliches Rechts y, en 1829, Die Geschichte der letztern Zeit 1814, vorzüglich in Rücksicht des öffenlichen Rechts, todos escritos mientras impartía las lecciones hegelianas sobre derecho. Leopold von Henning, quien también realizaría las lecciones legadas por Hegel entre 1825 y 1831, publicó incluso más que Gans sobre el tema: en 1823 escribió su propio manual sobre la filosofía hegeliana del derecho, Natur-und Staatsrecht, oder Philosophie des Rechts, nach Hegels "Grundlinien der Philosophie des Rechts", que comenzaría a trabajar desde 1824 en las lecciones. En 1825 publicó Ueber die verschiedenen Principien der Erkenntniß und Gültigkeit des Rechts. (Als Einleitung zu den Vorlesungen über Naturrecht), en 1826 Naturrecht oder Philosophie des Rechts, en 1829 su Geschichte des Preußischen Staats seit dem Anfange des 17ten Jahrhunderts y, más importante aún, publicó en 1828 su Politische 
Ökonomie oder National und-Staatswirtschaft, y en 1831 su Grundzüge der National-Ökonomie und der Staatswirtschaft. Henning, además de impartir las lecciones hegelianas, ofició desde 1827 de editor del Jahrbücher für wissenschaftliche Kritik fundado por Hegel, y anunciado en su llegada a Berlín a través de la misiva que trabajamos en el primer apartado de esta investigación.

\section{CONCLUSIÓN}

Podemos establecer que, pese a las dificultades historiográficas referentes al esclarecimiento de las influencias sobre los aspectos centrales de la economía política en Hegel, las implicancias de este estudio sobrepasan las intenciones meramente filológicas. Los resultados de esta lectura, nos dan a entender que el análisis hegeliano sobre la economía política tiene alcances sobre la comprensión de su propio sistema de relaciones, en la medida que influye en la estructura ética desarrollada tanto en el periodo de Heidelberg como en el de Berlín. La ilustración del debate suscitado sobre la economía política abre la posibilidad a la distinción epistemológica en Hegel de las implicancias técnicas respecto a la nueva economía y al cameralismo económico-político consuetudinario. El tratamiento del "joven” Hegel de Jena sobre las problemáticas contemporáneas de la economía política difieren de su obra "madura" tanto en el desarrollo conceptual, como en la influencia dentro de su propio sistema. Si en una época temprana Hegel podría haber sido perfectamente lector asiduo de Steuart y Smith, se nos hace difícil vislumbrar influencias cruciales en su etapa tardía para la construcción de su propia topología conceptual.

Sin embargo, es del todo plausible que la preocupación de Hegel por una dilucidación de la economía política sea base para la determinación de aspectos sociales y políticos que genera situaciones paradojales. La preocupación temprana por la eticidad en Hegel permite entender la sociedad comercial mercantil como fuente de consecuencias directas de pauperización de la población. La paleta conceptual que utilizará Hegel a lo largo de su obra para caracterizar los diferentes aspectos de la economía política (Nationalökonomie, Staatsökonomie, Politische Ökonomie y Staatswirtschaft) dan cuenta de los intentos por dotar de criterios epistemológicos a fenómenos que se derivan de la nueva economía política inglesa. Sin embargo, hacemos el énfasis que la pretensión crucial respecto a estos tópicos no estribaba en el discutir los pilares del modo de producción moderno, sino más bien derivar criterios filosóficos robustos que cumplan una función significativa dentro del marco histórico. La preferencia, en este contexto según la hipótesis expuesta por Rósza, de una Staatsökonomie por sobre una Staatswirtschaft en el vocabulario de Hegel abre la llave para comprender su boceto de economía política no como una discusión sobre principios y formas económicas, sino solo como un modo de representación del comportamiento sistémico de ésta en relación a la realidad contingente. 


\section{Referencias}

Aliscioni, C. (2010). El capital en Hegel. Estudio sobre la lógica económica de la Filosofía del Derecho. Rosario: Homo Sapiens.

Barcella, F. (1975). L'Antike in Hegel e altri scritti marxisti. Urbino: Argalia.

Chamley, P. (1962). Économie politique et philosophie chez Steuart et Hegel. Strasbourg: Dalloz.

DeGrood, D. (1979). Dialectics and Revolution. Amsterdam: Grüner.

DeTracy, D. (1823). Traité d'Économie politique. Paris: Bouguet et Lévi.

Dotti, J. (1983). Dialéctica y derecho. El proyecto ético-político hegeliano. Buenos Aires: Hachette.

Epstein, K. (1966). The Genesis of German Conservatism. Princeton: Princeton University Press.

Gans, E. (2005). Naturrecht und Universalrechtsgeschichte, Vorlesungen nach G. W. F. Hegel. Tübingen: Mohr Siebeck.

Harada, T. (1987). Politische Ökonomie des Idealismus und der Romantik. Korporativismus von Fichte, Müller und Hegel. Berlin: Duncker \& Homblot.

Hegel, G. W. F. (1969). Werke in 20 Bänden. Frankfurt: Shurkamp.

Hegel, G. W. F. (1973). Vorlesungen über Rechtsphilosophie 1818-1831. Herausgegeben von Karl-Heinz Ilting. 4 Bände. Sttutgart: Frommann-Holzboog.

Hegel, G. W. F. (1979). Sobre las maneras de tratar científicamente el derecho natural. Madrid: Aguilar.

Hegel, G. W. F. (1983). Vorlesungen über Naturrecht und Staatswissenschaft. Heidelberg 1817/18 mit Nachträgen aus der Vorlesung 1818/19. Nachgeschrieben von P. Wannenmann. Hamburg: Felix Meiner.

Hegel, G. W. F. (1992). El sistema de la eticidad. Madrid: Editorial Nacional.

Hegel, G. W. F. (1993). Fundamentos de la filosofía del derecho. Madrid: Libertarias.

Hegel, G. W. F. (1995). Lectures on Natural Right and Political Science: The First Philosophy of Right. Berkeley: University of California Press.

Hegel, G. W. F. (2003). Escritos de juventud. Madrid: FCE.

Hegel, G. W. F. (2006). Filosofía real. Madrid: FCE.

Henderson, J. \& Davis, J. (1991). Adam Smith's Influence on Hegel's Philosophical Writings. Journal of the History of Economic Thought, 13(2), 184-204.

Knortz, H. \& Laudenberg, B. (2014). Goethe, der Merkantilismus und die Inflation. Zum ökonomischen Wissen und Handeln Goethes und seiner Figuren. Berlin: Lit Verlag.

Lai, C. (2003). Adam Smith Across Nations. Translations and Receptions of The Wealth of Nations. Oxford: Oxford University Press.
Lukács, G. (1963). El joven Hegel y los problemas de la sociedad capitalista. México, D. F.: Grijalbo.

MacGregor, D. (1996). Hegel, Marx, and the English State. Toronto: University of Toronto Press.

Petry, M. (1976). Hegel and the 'Morning Chronicle'. HegelStudien, 11, 11-80.

Pinkard, T. (2002). Hegel. Una biografía. Madrid: Acento.

Plejánov, G. (2012). En el $60^{\circ}$ aniversario de la muerte de Hegel. Revista Colombiana de Sociología, 35(1), 153-175.

Priddat, B. (1990). Hegel als Ökonom. Berlin: Duncker \& Humblot.

Ricardo, D. (1971). Principles of political economy and taxation. London: Pelican Classics.

Rinaldi, G. \& Rossi T. (Eds.). (2010). Il pensiero di Hegel nell'Età della globalizzazione. Atti del Congreso Internazionale. Urbino, 3-5 giugno 2010. Roma: Arcane.

Rózsa, E. \& Quante, M. (Eds.). (2007). Hegels Konzeption praktische Individualität. Paderborn: Mentis Verlag.

Salomon, R. (1983). In the Spirit of Hegel: A Study of G. W. F. Hegel's Phenomenology of Spirit. Oxford: Oxford University Press.

Say, J. (2001). Tratado de economía política. México, D. F.: FCE. Smith, A. (1794). Untersuchung über die Natur und die Ursachen des Nationalreichthums. Breslau: G. W. Korn.

Smith, A. (1981). An Inquiry into the Nature and Causes of the Wealth of Nations. Indianapolis: Liberty Fund.

Smith, A. (2004). La riqueza de las naciones. Madrid: Alianza.

Steuart, J. (1767). Principles of Political Economy. London: A. Millar \& T. Cadell.

Tortajada, R. (1999). The Economics of James Steuart. London: Routledge.

Tribe, K. (1988). Governing Economy. The Reformation of German Economic Discourse 1750-1840. Cambridge: Cambridge University Press.

Waszek, N. (1984). Hegels Schottische Bettler. Hegel-Studien, 19, 311-316.

Waszek, N. (1985). Hegels Exzerpte aus der 'Edinburgh Review' 1817-1819. Hegel-Studien, 20, 79-112.

Waszek, N. (1986). Hegels Exzerpte aus der 'Quarterly Review' 1817-1819. Hegel-Studien, 21, 9-25.

Waszek, N. (1988). The Scottish Enlightenment and Hegel's Account of 'Civil Society'. Dordrecht: Kluwer Academic Publishers.

Waszek, N. (2011). O estatuto da economía política na filosofía prática de Hegel. Revista Opinião Filosófica, 1(2), 56-72. 